\title{
Simulation of Performance Response of State Variables of a Chemical Boiler Process
}

\author{
Donatus O. Njoku ${ }^{\# 1}$, Asagba P. O. ${ }^{* 2}$, Chilaka U. Longinus ${ }^{\# 3}$, Amaefule I. A. ${ }^{\# 3}$, Igwe S. Onyema ${ }^{\# 4}$ \\ ${ }^{\# 1}$ Department of Computer Science, Federal University of Technology Owerri, Nigeria \\ ${ }^{\# 2}$ Department of Computer Science, University of Port Harcourt, Nigeria \\ ${ }^{\# 3}$ Department of Computer Science, Imo State University, Owerri, Nigeria \\ ${ }^{\# 4}$ Department of Computer Science, Federal Polytechnic Nekede, Owerri, Nigeria
}

\begin{abstract}
This paper has presented simulation of performance response of state variables of a chemical boiler process. The transfer function of a boiler flow control process of a chemical plant was obtained. The transfer function was transformed into state space form to study the state variables of the system. An optimal regulator was designed using MATLAB programme. The developed optimal regulator was added to the loop of the system to form a closed loop system. A Simulink model was developed and used to study performance response of the system. Simulation was carried out for two conditions, open loop and closed loop. The simulation results indicated that the performance responses of the state variables were improved and better stability achieved with the inclusion of the designed feedback gain matrix of the optimal regulator.
\end{abstract}

Keywords-Boiler, Optimal regulator, State variable, Feedback gain matrix.

\section{INTRODUCTION}

In the analysis and of control systems, the dynamics of the systems are often represented in the form of transfer functions. Though the transfer function model provides simple and powerful analysis and design techniques [1], it suffers from certain drawbacks, for example, it is only defined under zero initial conditions [1]. Its application is limited to linear time invariant (LTI) systems, and it is generally restricted to single-inputsingle-output (SISO) systems due to the fact that this method becomes highly complex for use in multi-inputmulti-output (MIMO) systems [1]. Also, in transfer function technique, only the system output is revealed for a given input without no information about the internal state of the system.

The mathematical equations describing the characteristics of a physical system can be represented using the state variables equation. However, the concept of state variable is not only applicable to the analysis of physical systems but also used in analysing biological, social and economic systems [2][3]. State variable formulation has been made possible using mathematical modelling of a system which, with the output, gives information on the state variables at a specified points along the flow of signals [3]. State variable is a time domain technique that provides a basis for modern control theory and system optimization [3]. It serves as a powerful technique for analysis and design of linear, and nonlinear, time invariant or time varying MIMO systems [1] [3]. Despite the merits of state variable analysis, the transfer function techniques provide the control engineer an insight into the physical state of the system and largely support in pilot design of system. In this case, a complex system is approximated and represented by more simplified and manageable model [3].

The objective of this paper is to design an optimal regulator that will improve state variable performance response of a chemical plant. Simulation is carried out in MATLAB/Simulink to ascertain the effectiveness of the design matrix gain of the optimal regulator.

\section{LITERATURE REVIEW}

The state of a system is a set of variables whose knowledge and the input functions will, in addition with the dynamic equations describing the system, determine the future state and output of the system. These set of variables are called state variables. For a given dynamic system, the state of such system can be represented in terms of a set of states variables as shown in Fig. 1. 


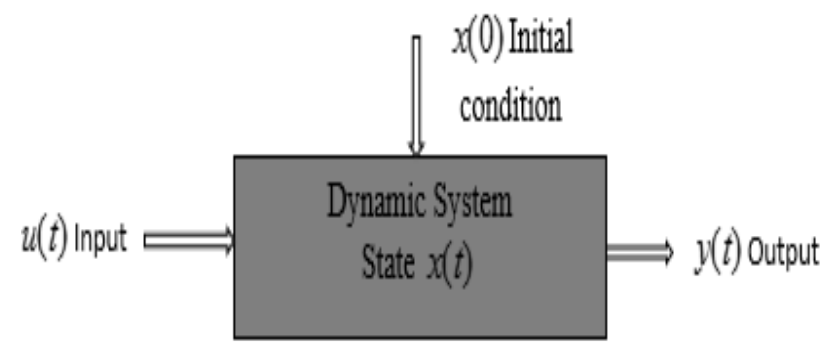

Fig. 1 state variable of a dynamic system

Generally, the system state variables describe the response of a system in future, provided the initial state, the inputs (excitation), and the dynamic equations describing the system are known. A state space equation can be formulated from state space analysis of three variables of interest: input variables, output variables, and state variables.

\section{A. State Space Equation}

A linear state space system can be represented in the form given by [4] in Eq. (1):

$\&=A x+B u$

$y=C x+D u$

where $A, B, C$ and $D$ are the constant matrices such that $\mathrm{A}$ is the state matrix, $\mathrm{B}$ is the input matrix, $\mathrm{C}$ is the output matrix, and Dis the direct transition matrix.

\section{B. Problem Formulation}

Chemical (Power) plant boiler is a complex industrial equipment [5]. Considering the nonlinear behaviour of such system, a significant modelling and simulation challenge is experienced. A significant work on boiler modelling has been done before now including validation with reference plant [5]. Modelling and simulation has a key role in industry and process development [6]. According to Shoorabeh et al [6], two methods of modelling exist. I). the method based on dynamic behaviour and relation of variables and states in physical equations and consequent differential equations from the real system (mathematical modelling) [6]. II). the method based on input-output data and expertise or knowledge of the system (Empirical Modelling) [6, 7]. According to Rusinowski and Stanek [8], empirical (or experimental) modelling is easier to implement and to design for future development and changes. In addition, Angell et al [9] declared that setup of data gathering and communication is essential for empirical modelling.

A boiler of a chemical plant is taken as a case study [10]. The role of a boiler is to deliver steam with an established or known pressure and temperature to a turbine or other process equipment [6]. It is required to study the state variable performance response of the process temperature. The mathematical model of the process is obtained using experimental data available and transfer function of the system [10] is given by Eq. (2):

$G(s)=\frac{5(s+1)}{s(s+1)(s+6)}$

\section{III.STATE VARIABLE FORMULATION}

The transfer function given in Eq. (2) is transformed into its equivalent state space form as follows: Equation (2) is represented in Eq. (3) as follows using the approach in [3, 11].

$\frac{X(s)}{U(s)} \times \frac{Y(s)}{X(s)}=\frac{5 s+5}{s^{3}+7 s^{2}+6 s}$

then,

$\frac{X(s)}{u(s)}=\frac{1}{s^{3}+7 s^{2}+6 s}$

Assuming zero initial conditions:

$s^{3} X(s)+7 s^{2} X(s)+6 s X(s)=u(s)$

Let 
$x=x_{1}$

$\&=x_{2}$

$\varkappa_{2}=x_{3}$

\& $=-7 x_{3}-6 x_{2}+u(t)$

Transforming Eq. (6) to (9) to state space form, gives:

$\left[\begin{array}{l}\& \\ \& \\ \&\end{array}\right]=\left[\begin{array}{ccc}0 & 1 & 0 \\ 0 & 0 & 1 \\ 0 & -6 & -7\end{array}\right]\left[\begin{array}{l}x_{1} \\ x_{2} \\ x_{3}\end{array}\right]+\left[\begin{array}{l}0 \\ 0 \\ 1\end{array}\right] u(t)$

Also,

$$
\begin{aligned}
& \frac{Y(s)}{X(s)}=5 s+5 \\
& Y(s)=5 s X(s)+5 X(s)
\end{aligned}
$$

Solving Eq. (12) and transforming it into state space form yields:

$$
y=\left[\begin{array}{lll}
5 & 5 & 0
\end{array}\right]\left[\begin{array}{l}
x_{1} \\
x_{2} \\
x_{3}
\end{array}\right]
$$

In this paper, $\mathrm{D}=0$.

In this paper, the concept of optimal regulator is adopted to improve the performance response of state variables of a chemical plant boiler. The optimal regulator design is achieved with state matrix $A$ and output matrix $B$. The matrices $Q$ and $R$ which are real symmetric matrices are obtained as stated in Eq. (14) and (15).

$Q=\left[\begin{array}{ccc}100 & 0 & 0 \\ 0 & 1 & 0 \\ 0 & 0 & 1\end{array}\right]$

$R=[0.01]$

With the application of MATLAB, the command represented by Eq. (16) is used to obtain the feedback matrix K.

$K=\operatorname{lqr}(A, B, Q, R)$

Running the programme in Eq. (16), the resulting Eq. (17) gives the gains $k_{1}, k_{2}, k_{3}$ of the feedback gain matrix $K$ given by:

$K=\left[\begin{array}{lll}100 & 51.5454 & 8.8774\end{array}\right]$

\section{A. Open loop Simulink Model}

In the open loop case, only the $A, B, C$ and $D$ matrices are represented in the Simulink state space model as shown in Fig. 2.

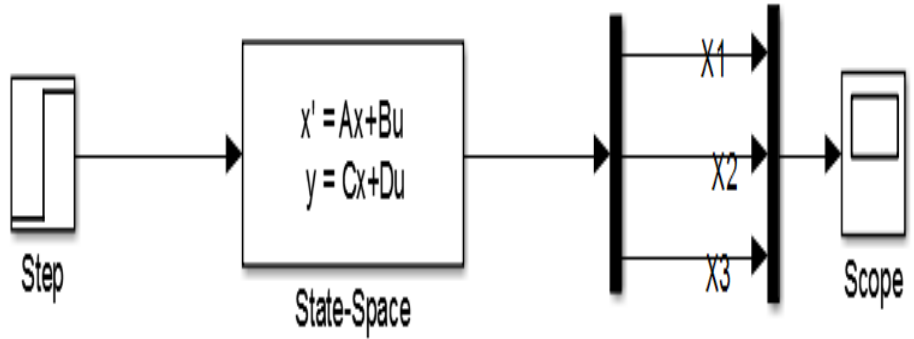

Fig.2 State space Simulink model for open loop 


\section{B. Closed loop Simulation}

The closed loop Simulink model is shown in Fig. 3. In this case, the $K$ matrix is included in the feedback loop.

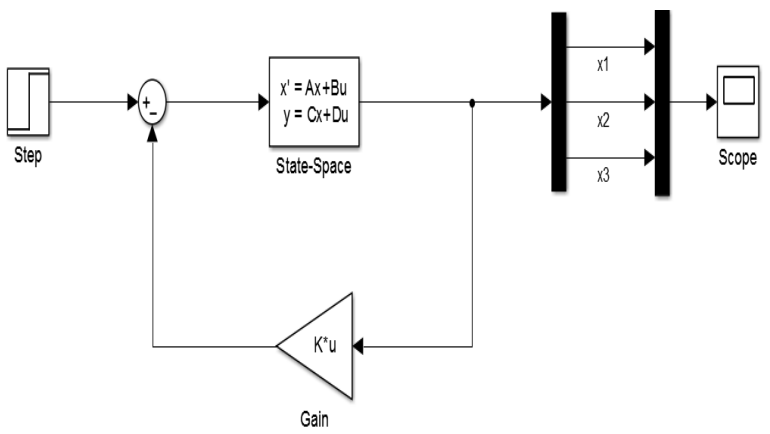

Fig. 3 Simulink model with feedback matrix

\section{IV.SIMULATION RESULT AND DISCUSSION}

The simulations results obtained are presented in Fig. 4 and 5. The open loop simulation result is shown in Fig. 4 and Fig. 5 represents the closed loop result.

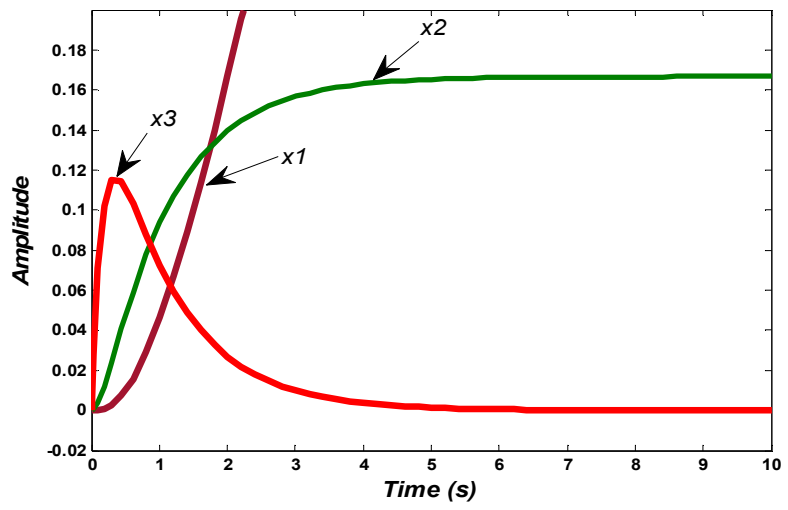

Fig. 4 Open loop performance response

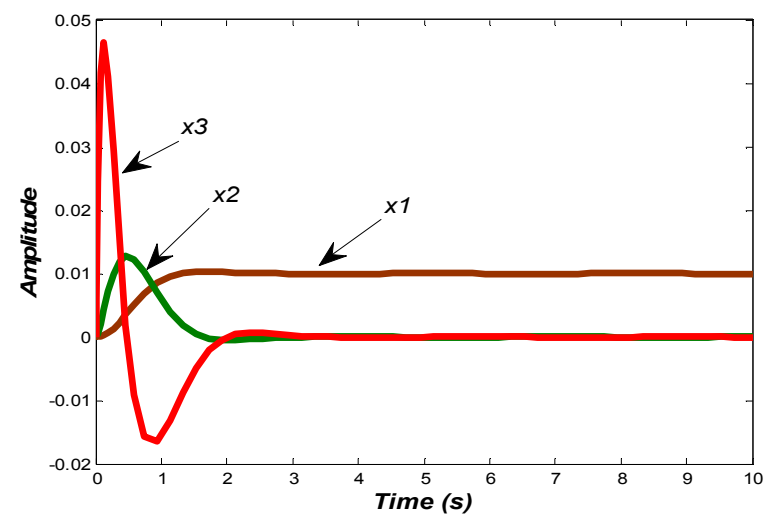

Fig. 5 Closed loop performance response 
The open loop result shown in Fig. 4 represents performance response of the state variables of a boiler chemical plant. The result shows that performance response of the state variable requires improvement and increase stability. The focus is on designing an optimal feedback regulator that will improve the stability of the performance response. A feedback regulator of gain matrix $K$ is designed using the concept of linear quadratic regulator, and added to the closed loop. The performance response obtained from the simulation in MATLAB/Simulink environment as shown in Fig. 5 indicated that the system state variable response to step input is largely improved.

\section{Conclusion}

An optimal regulator system has been designed in this paper to improve the response performance of state variables of a boiler chemical plant. The transfer function of a boiler process temperature was obtained and then transform into state space form. The state space model is developed to study the dynamic response of the variables. A MATLAB programme was used to implement the feedback gain matrix, $K$. Open loop and closed loop simulations were carried out to study the response performance of the set of state variables of the system during uncompensated and compensated conditions.

\section{REFERENCES}

[1] I. J. Nagrath, and M. Gopal, “Control Systems Engineering,” New Age International Publishers, 4th Edition, pp. 570-640, 2005.

[2] JC.D. Richard, and R.H., Bishop, "Modern Control Systems," Prentice Hall, Upper Saddle River, NJ, 12 th Edition, 2011.

[3] D.O. Njoku, C. E. Nwokorie, and A.I. Amaefule, "Simulation of State Variable Compensator for Linearized Mechanical System," International Journal of Research, vol. 5 (20), pp 452-454, September 2018

[4] Anonymous, "Aircraft Pitch: Simulink Modeling, Control Tutorials for MATLAB and SIMULINK" ctms.engin.umich.edu/CTMS/index.php?example=AircraftPitch\&section=SimulinkModeling

[5] P. U. Sunnil, J. Barve, and P.S.V. Nataraj, "Boiler model and simulation for control design and validation," Third International Conference on Advances in Control and Optimization of Dynamic Systems, pp 936-940, March 13-15, 2014.

[6] J. Shoorabeh, F. Shabaninia, and I. Karimi, "Industrial Boiler Modelling and Control Based on Adaptive Neuro Fuzzy Inference System and Implementation in S7-400H PLC," International Journa of Review in Life Sciences, vol. 5 (3), pp 40-48, 2015.

[7] R. Boyatt, A. Harfield, and M. Beynon, "Learning about and through Empirical modelling,” ICALT, pp 662-666, 2006.

[8] H. Rusinowski and W. Stanek, “Neural modelling of steam boilers,” Energy Conversation Management, vol. 48, pp 2802-2809, 2007.

[9] C. Angell, P. M. Kind, E. Henriksen, and Q. Guttersued, “An empirical Mathematical modelling approach to upper secondary physics," Physics Education, 43 (3), pp 256-264, 2008;

[10] R. Malhotra and R. Sodhi, “Boiler Flow Control Using PID and Fuzzy Logic Controller,” IJCSET, vol. 1 (6), pp 315-319, 2011.

[11] S. Hasan Saeed, Automatic "Control Systems (with MATLAB PROGRAMS)," S.K. Katana and Sons, Darya Ganj, New Delhi, 7th Revised Edition, January, 2012 